\title{
Opinion Summarization in Bengali: A Theme Network Model
}

\author{
Amitava Das ${ }^{1}$ and Sivaji Bandyopadhyay ${ }^{2}$ \\ Department of Computer Science and Engineering \\ Jadavpur University, Kolkata 700032, India \\ amitava.santu@gmail.com ${ }^{1}$ and sivaji_cse_ju@yahoo.com ${ }^{2}$
}

\begin{abstract}
Theme network is a semantic network of document specific themes. So far Natural Language Processing (NLP) research patronized much of topic based summarizer system, unable to capture thematic semantic affinity of any text i.e. a news article containing the concepts, "gun," "convenience store," "demand money" and "make getaway" might suggest the topics "robbery" and "crime". In this paper the development of an opinion summarization system that works on Bengali News corpus has been described. The system identifies the sentiment information in each document, aggregates them and represents the summary information in text. The present system follows a topic-sentiment model for sentiment identification and aggregation. Topic-sentiment model is designed as discourse level theme identification and the topic-sentiment aggregation is achieved by theme clustering (k-means) and Document level Theme Relational Graph representation. The Document Level Theme Relational Graph is finally used for candidate summary sentence selection by standard page rank algorithms used in Information Retrieval (IR). As Bengali is a resource constraint language, the building of annotated gold standard corpus and acquisition of linguistics tools for lexico-syntactic, syntactic and discourse level features extraction are described in this paper. The reported accuracy of the Theme detection technique is 83.60\% (precision), $\mathbf{7 6 . 4 4 \%}$ (recall) and $\mathbf{7 9 . 8 5 \%}$ (F-measure). The summarization system has been evaluated with Precision of $\mathbf{7 2 . 1 5 \%}$, Recall of $67.32 \%$ and F-measure of $69.65 \%$.
\end{abstract}

Keywords-Opinion Summarization, Topic-Sentiment, Theme, Topic based Opinion Summarization.

\section{INTRODUCTION}

The Web has become a rich source of various opinions in the form of product reviews, travel advice, social issue discussions, consumer complaints, movie review, stock market predictions, real estate market predictions, etc. Present computational systems must have to extend the power of understanding the sentiment/opinion expressed in an electronic text to act properly in society rather only deal with the topic of a document. The topic-document model of information retrieval has been studied for a long time and systems are available publicly since last decade. On the contrary Opinion Mining/Sentiment Analysis is still an unsolved research problem. Although a few systems like Bing ${ }^{1}$, Twitter Sentiment Analysis Tool ${ }^{2}$ are available in World Wide Web

\footnotetext{
${ }^{1}$ http://www.bing.com/

${ }^{2} \mathrm{http}: / /$ twittersentiment.appspot.com/
}

since last few years but still more research efforts are necessary to match the user satisfaction level and social need.

Researchers have taken multiple approaches towards the problem of Opinion Summarization like Topic-sentiment model, Textual summaries at single document or multiple document perspective and graphical summaries or visualization. The works on opinion tracking systems have explicitly incorporated temporal dimension. The topicsentiment model is well established for opinion retrieval.

The concept of reputation system was first introduced by [1]. The need of reputation system for both buyers and sellers was established to earn each other's trust in online interactions.

$\mathrm{Ku}$ et al., 2005 [2] selects representative words from a document set to identify the main concepts in the document set. A term is considered to represent a topic if it appears frequently across documents or appears frequently in each document. Different methodologies have been used to assign weights to each word both at document level and paragraph level. The precision and recall values of the system have been reported as 0.56 and 0.85 .

Zhou et al., 2006 [3] have proposed the architecture for generative summary from blogosphere. Typical multidocument summarization (MDS) systems focus on content selection followed by synthesis by removing redundancy across multiple input documents. The online discussion summarization system [3] work on an online discussion corpus where multiple participants are involved and discussion topics are being passed back and forth by various participants. MDS systems are insufficient in representing this aspect of the interactions. Due to the complex structure of the dialogue, similar subtopic structure identification in the participantwritten dialogues is essential. Maximum Entropy Model (MEMM) and Support Vector Machine (SVM) have been used with a number of relevant features.

Carenini et al., 2006 [4] present and compare two approaches to the task of multi document opinion summarization on evaluative texts. The first is a sentence extraction based approach while the second one is a natural language generation-based approach. Relevant extracted features are categorized in two types: User Defined Features (UDF) and Crude Features (CF) as described in [5]. The summary generation technique uses the aggregation of the extracted features, CF and UDF. Opinion aggregation has been 
done by the two relevant features: opinion strength and polarity. A new opinion distribution function feature has been introduced to capture the overall opinion distributed in corpus.

Kawai et al., 2007 [6] developed a news portal site called Fair News Reader (FNR) that recommends news articles with different sentiments for a user in each of the topics in which the user is interested. FNR can detect various sentiments of news articles and determine the sentimental preferences of a user based on the sentiments of previously read articles by the user. News articles collected from various news sites by crawling through them are stored in a database. The contents are integrated as needed and the summary is presented on one page. A sentiment vector on the basis of word lattice model has been generated for every document. A user sentiment model has been proposed based on user sentiment state. The user sentiment state model works on browsing history of the user. The intersection of the documents under User Vector and Sentiment Vector are presented as the result.

\section{RESOURCE ORGANIZATION}

Resource acquisition is one of the most challenging obstacles to work with resource constrained languages like Bengali. Bengali is the sixth ${ }^{3}$ popular language in the World, second in India and the national language in Bangladesh. Extensive NLP research activities in Bengali have started recently but resources like annotated corpus, various linguistic tools are still unavailable for Bengali. The manual annotation of gold standard corpus and acquisition of various tools used in the feature extraction for Bengali are described in this section.

\section{A. Gold Standard Data Acquisition}

\section{1) Corpus:}

For the present task a Bengali news corpus has been used that is developed from the archive of a leading Bengali news paper available on the Web. A portion of the corpus from the editorial pages, i.e., Reader's opinion section or Letters to the Editor Section containing $28 \mathrm{~K}$ word forms has been manually annotated with sentence level subjectivity and discourse level theme words. Detailed reports about this news corpus development in Bengali can be found in [7] reported in Table I below.

TABLE I. BENGALI CoRPus StATISTics

\begin{tabular}{|l|l|}
\hline \multicolumn{1}{|c|}{ Statistics } & \multicolumn{1}{c|}{ Size } \\
\hline Total number of documents & 100 \\
\hline Total number of sentences & 2234 \\
\hline Avgerage number of sentences in a document & 22 \\
\hline Total number of wordforms & 28807 \\
\hline Avgerage number of wordforms in a document & 288 \\
\hline Total number of distinct wordforms & 17176 \\
\hline
\end{tabular}

\section{2) Annotation}

http://en.wikipedia.org/wiki/List_of_languages_by_number_o f_native_speakers
From the collected document set (Letters to the Editor Section), some documents have been chosen for the annotation task. Documents that appeared within an interval of four months are chosen on the hypothesis that these letters to the editors will be on related events. A simple annotation tool has been designed for annotating the sentences considered to be important for opinion summarization. Three annotators (Mr. X, Mr. Y and Mr. Z) participated in the present task.

Annotators were asked to annotate sentences for summary and to mark the theme words (topical expressions) in those sentences.

The annotation tool highlights the sentiment words $[8]^{4}$ by four different colors within a document according to their POS categories (Noun, Adjective, Adverb and Verb). This technique helps to increase the speed of annotation process. Finally 100 annotated documents have been developed.

\section{3) Inter-annotator Agreement}

The agreement of annotations among three annotators has been evaluated. The agreements of tag values at theme words level and sentence levels are listed in Tables II and III respectively.

TABLE II. AGREEMENT OF ANNOTATORS AT THEME WORDS LEVEL

\begin{tabular}{|l|c|c|c|c|}
\hline Annotators & X vs. Y & X Vs. Z & Y Vs. Z & Avg \\
\hline Percentage & $82.64 \%$ & $71.78 \%$ & $80.47 \%$ & $78.30 \%$ \\
\hline All Agree & \multicolumn{5}{|c|}{$69.06 \%$} \\
\hline
\end{tabular}

TABLE III. AGREEMENT OF ANNOTATORS AT SENTENCE LEVEL

\begin{tabular}{|l|c|c|c|c|}
\hline Annotators & X vs. Y & X Vs. Z & Y Vs. Z & Avg \\
\hline Percentage & $73.87 \%$ & $69.06 \%$ & $60.44 \%$ & $67.8 \%$ \\
\hline All Agree & \multicolumn{5}{|c|}{$58.66 \%$} \\
\hline
\end{tabular}

From the analysis of inter-annotator agreement, it is observed that the agreement drops fast as the number of annotator increases. It is less possible to have consistent annotations when more annotators are involved. In the present task the inter-annotator agreement is better for theme words annotation rather than candidate sentence identification for summary though a small number of documents have been considered.

Further discussion with annotators reveals that the psychology of annotators is to grasp as many as possible theme words identification during annotation but the same groups of annotators are more cautious during sentence identification for summary as they are very conscious to find out the most concise set of sentences that best describe the opinionated snapshot of any document. The annotators were working independent of each other and they were not trained linguists.

\section{B. Subjectivity Classifier}

Work in opinion mining and classification often assumes the incoming documents to be opinionated. Opinion mining system makes false hits while attempting to summarize non-

\footnotetext{
${ }^{4}$ http://www.amitavadas.com/sentiwordnet.php
} 
subjective or factual sentences or documents. It becomes imperative to decide whether a given document contains subjective information or not as well as to identify which portions of the document are subjective or factual. This task is termed as subjectivity detection in sentiment literature. The subjectivity classifier that uses CRF machine learning technique and described in [7] has been used here. The recall measure of the present classifier is greater than its precision value. The evaluation results of the classifier are $72.16 \%$ (Precision) and 76.00 (recall) on the News Corpus.

\section{Feature Organization}

The set of features used in the present task have been categorized as Lexico-Syntactic, Syntactic and Discourse level features. These are listed in the Table IV below and have been described in the subsequent subsections.

\begin{tabular}{|l|l|}
\multicolumn{1}{|c}{ TABLE IV. FEATURES } \\
\hline \multirow{4}{*}{ Types } & \multicolumn{1}{c}{ Features } \\
\hline \multirow{4}{*}{ Lexico-Syntactic } & POS \\
\cline { 2 - 2 } & SentiWordNet \\
\cline { 2 - 2 } Syntactic & Frequency \\
\cline { 2 - 2 } & Stemming \\
\hline \multirow{4}{*}{ Discourse Level } & Chunk Label \\
\cline { 2 - 2 } & Dependency Parsing Depth \\
\cline { 2 - 2 } & Title of the Document \\
\cline { 2 - 2 } & First Paragraph \\
\cline { 2 - 2 } & Term Distribution \\
\cline { 2 - 2 } & Collocation \\
\hline
\end{tabular}

\section{1) Lexico-Syntactic Features}

\section{a) Part of Speech (POS)}

It has been shown by [9], [10] etc. that opinion bearing words in sentences are mainly adjective, adverb, noun and verbs. Many opinion mining tasks, like [11] are mostly based on adjective words. Further details of development of POS tagging system could be found in [12].

\section{b) SentiWordNet (Bengali)}

Words that are present in the SentiWordNet carry opinion information. The developed SentiWordNet (Bengali) [8] is used as an important feature during the learning process. Words which are collected directly from SentiWordNet (Bengali) are tagged with positivity or negativity score. The subjectivity score of these words are calculated as:

$$
E_{s}=\left|S_{p}\right|+\left|S_{n}\right|
$$

where $E_{s}$ is the resultant subjective measure and $S_{p}, S_{n}$ are the positivity and negativity score respectively.

\section{c) Frequency}

Frequency always plays a crucial role in identifying the importance of a word in the document. The system generates four separate high frequent word lists after function words are removed for four POS categories: Adjective, Adverb, Verb and
Noun. Word frequency values are then effectively used as a crucial feature in the Theme Detection technique.

\section{d) Stemming}

Several words in a sentence that carry opinion information may be present in inflected forms. Stemming is necessary for such inflected words before they can be searched in appropriate lists. Due to non availability of good stemmers in Indian languages especially in Bengali, a stemmer based on stemming cluster technique has been used as described in [13]. This stemmer analyzes prefixes and suffixes of all the word forms present in a particular document. Words that are identified to have the same root form are grouped in a finite number of clusters with the identified root word as cluster center.

\section{2) Syntactic Features}

\section{a) Chunk Label}

Chunk level information is effectively used as a feature in supervised classifier. Chunk labels are defined as B-X (Beginning), I-X (Intermediate) and E-X (End), where $\mathrm{X}$ is the chunk label. In the task of identification of Theme expressions, chunk label markers play a crucial role. Further details of development of chunking system could be found in [12].

\section{b) Dependency Parser}

Dependency depth feature is very useful to identify Theme expressions. A particular Theme word generally occurs within a particular range of depth in a dependency tree. Theme expressions may be a Named Entity (NE: person, organization or location names), a common noun (Ex: accident, bomb blast, strike etc) or words of other POS categories. It has been observed that depending upon the nature of Theme expressions it can occur within a certain depth in the dependency tree for the sentence. A statistical dependency parser has been used for Bengali as described in [13].

\section{3) Discourse Level Features}

\section{a) Positional Aspect}

Depending upon the position of the thematic clue, every document is divided into a number of zones. The features considered for each document are Title words of the document, the first paragraph words and the words from the last two sentences. A detailed study was done on the Bengali news corpus to identify the roles of the positional aspect features of a document (first paragraph, last two sentences) in the detection of theme words and subjective sentences for generating the summary of the document. The importance of these positional features on the gold standard set prepared for Bengali is shown in Tables $\mathrm{V}$.

\section{b) Title Words}

It has been observed that the Title words of a document always carry some meaningful thematic information. The title word feature has been used as a binary feature during CRF based machine learning.

\section{c) First Paragraph Words}

People usually give a brief idea of their beliefs and speculations in the first paragraph of the document and subsequently elaborate or support their ideas with relevant 
reasoning or factual information. Hence first paragraph words are informative in the detection of Thematic Expressions.

\section{d) Words From Last Two Sentences}

It is a general practice of writing style that every document concludes with a summary of the opinions expressed in the document.

TABLE V. Statistics on Positional Aspect.

\begin{tabular}{|l|l|}
\hline Positional Factors & Bengali \\
\hline First Paragraph & $56.80 \%$ \\
\hline Last Two Sentences & $78.00 \%$ \\
\hline
\end{tabular}

\section{e) Term Distribution Model}

An alternative to the classical TF-IDF weighting mechanism of standard IR has been proposed as a model for the distribution of a word. The model characterizes and captures the informativeness of a word by measuring how regularly the word is distributed in a document. As discussed in Section I [4] introduced the opinion distribution function feature to capture the overall opinion distributed in the corpus. Thus the objective is to estimate $f_{d}\left(w_{i}\right)$ that measures the distribution pattern of the $\mathrm{k}$ occurrences of the word $w_{i}$ in a document $d$. Zipf's law describes distribution patterns of words in an entire corpus. In contrast, term distribution models capture regularities of word occurrence in subunits of a corpus (e.g., documents, paragraphs or chapters of a book). A good understanding of the distribution patterns is useful to assess the likelihood of occurrences of a word in some specific positions (e.g., first paragraph or last two sentences) of a unit of text. Most term distribution models try to characterize the informativeness of a word identified by inverse document frequency (IDF). In the present work, the distribution pattern of a word within a document formalizes the notion of topicsentiment informativeness. This is based on the Poisson distribution. Significant Theme words are identified using TF, Positional and Distribution factor. The distribution function for each theme word in a document is evaluated as follows:

$$
f_{d}\left(w_{i}\right)=\sum_{i=1}^{n}\left(S_{i}-S_{i-1}\right) / n+\sum_{i=1}^{n}\left(T W_{i}-T W_{i-1}\right) / n
$$

where $n=$ number of sentences in a document with a particular theme word $\mathrm{S}_{\mathrm{i}}=$ sentence id of the current sentence containing the theme word and $\mathrm{S}_{\mathrm{i}-1}=$ sentence id of the previous sentence containing the query term, $T W_{i}$ is the positional id of current Theme word and $T W_{i-1}$ is the positional id of the previous Theme word.

\section{f) Collocation}

Collocation with other thematic word/expression is undoubtedly an important clue for identification of theme sequence patterns in a document. A window size of 5 including the present word is considered during training to capture the collocation with other thematic words/expressions.

\section{THEME DETECTION}

Term Frequency (TF) plays a crucial role to identify document relevance in Topic-Based Information Retrieval. The motivation behind developing Theme detection technique is that in many documents relevant words may not occur frequently or irrelevant words may occur frequently. Moreover for sentiment analysis topic words should have sentiment conceptuality. The Theme detection technique has been proposed to resolve these issues to identify discourse level most relevant topic-semantic nodes in terms of word or expressions using a standard machine learning technique. The machine learning technique used here is Conditional Random Field $(\mathrm{CRF})^{5}$. The theme word detection is defined as a sequence labeling problem. Depending upon the series of input feature, each word is tagged as either Theme Word (TW) or Other $(\mathrm{O})$

\section{THEME CLUSTERING}

Theme clustering algorithms partition a set of documents into finite number of topic based groups or clusters in terms of theme words/expressions. The task of document clustering is to create a reasonable set of clusters for a given set of documents. A reasonable cluster is defined as the one that maximizes the within-cluster document similarity and minimizes betweencluster similarities. There are two principal motivations for the use of this technique in theme clustering setting: efficiency, and the cluster hypothesis.

The cluster hypothesis [14] takes this argument a step further by asserting that retrieval from a clustered collection will not only be more efficient, but will in fact improve retrieval performance in terms of recall and precision. The basic notion behind this hypothesis is that by separating documents according to topic, relevant documents will be found together in the same cluster, and non-relevant documents will be avoided since they will reside in clusters that are not used for retrieval. Despite the plausibility of this hypothesis, there is only mixed experimental support for it. Results vary considerably based on the clustering algorithm and document collection in use [15].

Application of the clustering technique to the three sample documents results in the following theme-by-document matrix, A, where the rows represent Docl, Doc7 and Doc13 and the columns represent the themes politics, sport, and travel.

$$
A=\left[\begin{array}{ccc}
\text { election } & \text { cricket } & \text { hotel } \\
\text { parliament } & \text { sachin } & \text { vacation } \\
\text { governor } & \text { soccer } & \text { tourist }
\end{array}\right]
$$

The similarity between vectors is calculated by assigning numerical weights to these words and then using the cosine similarity measure as specified in the following equation.

$$
s\left(\vec{q}_{k}, \overrightarrow{d_{j}}\right)=\vec{q}_{k} \cdot \vec{d}_{j}=\sum_{i=1}^{N} w_{i, k} \times w_{i, j}--(1)
$$

\footnotetext{
${ }^{5}$ http://crfpp.sourceforge.net
} 
This equation specifies what is known as the dot product between vectors. Now, in general, the dot product between two vectors is not particularly useful as a similarity metric, since it is too sensitive to the absolute magnitudes of the various dimensions. However, the dot product between vectors that have been length normalized has a useful and intuitive interpretation: it computes the cosine of the angle between the two vectors. When two documents are identical they will receive a cosine of one; when they are orthogonal (share on common terms) they will receive a cosine of zero. Note that if for some reason the vectors are not stored in a normalized form, then the normalization can be incorporated directly into the similarity measure as follows.

$$
s\left(\overrightarrow{q_{k}, d_{j}}\right)=\frac{\sum_{i=1}^{N} w_{i, k} \times w_{i, j}}{\sqrt{\sum_{i=1 w i, k}^{N} \times \sqrt{\sum_{i=1 w i, k}^{N}}}}---(2)
$$

Of course, in situations where the document collection is relatively static, it makes sense to normalize the document vectors once and store them, rather than include the normalization in the similarity metric.

Calculating the similarity measure and using a predefined threshold value, documents are classified using standard bottom-up soft clustering k-means technique. The predefined threshold value is experimentally set as 0.5 as shown in Table VI.

$$
\begin{aligned}
& 1 \text { Given: a set } X=\left\{\overrightarrow{x_{1}}, \ldots ., \overrightarrow{x_{n}}\right\} \subseteq R^{m} \\
& 2 \text { a distance measure } d: R^{m} \times R^{m} \rightarrow R \\
& 3 \text { a function for computing the mean } \\
& \mu: P(R) \rightarrow R^{m} \\
& 4 \text { Select } k \text { initial centers } \vec{f}_{1}, \ldots, \vec{f}_{k} \\
& 5 \text { while stopping criterion is not true do } \\
& 6 \text { for all clusters } c_{j} \text { do } \\
& 7 \\
& c_{j}=\left\{\vec{x}_{i} \mid \forall \vec{f}_{i} d\left(\vec{x}_{i}, \vec{f}_{j}\right) \leq d\left(\left(\overrightarrow{x_{i}}, \overrightarrow{f_{i}}\right)\right)\right\} \\
& 8 \text { end } \\
& 9 \text { for all means } \vec{f}_{j} \text { do } \\
& 10 \quad \vec{f}_{j}=\mu\left(c_{j}\right) \\
& 11 \\
& 12 \text { end }
\end{aligned}
$$

\begin{tabular}{|c|c|c|c|c|c|c|}
\hline \multicolumn{7}{|c|}{ Generated Clusters } \\
\hline ID & Themes & 1 & 2 & 3 & 4 & 5 \\
\hline 1 & $\begin{array}{l}\text { প्रশাসन } \\
\text { (administration) }\end{array}$ & 0.63 & 0.12 & 0.04 & 0.09 & 0.11 \\
\hline 1 & $\begin{array}{l}\text { সুশাসন (good- } \\
\text { government) }\end{array}$ & 0.58 & 0.11 & 0.06 & 0.10 & 0.14 \\
\hline 1 & সমাজ ( Society ) & 0.58 & 0.12 & 0.03 & 0.10 & 0.17 \\
\hline 1 & আইন (Law) & 0.55 & 0.14 & 0.08 & 0.08 & 0.15 \\
\hline 2 & গবেষণা (Research ) & 0.11 & 0.59 & 0.02 & 0.14 & 0.15 \\
\hline 2 & কলেজ (College) & 0.15 & 0.55 & 0.01 & 0.13 & 0.16 \\
\hline 2 & $\begin{array}{l}\text { উष্চশিক্ষা } \quad \text { (Higher } \\
\text { Study) }\end{array}$ & 0.12 & 0.66 & 0.01 & 0.09 & 0.11 \\
\hline 3 & জেহাদি ( Jehadi ) & 0.13 & 0.05 & 0.58 & 0.09 & 0.16 \\
\hline 3 & মসজিদ (Mosque) & 0.05 & 0.01 & 0.86 & 0.02 & 0.06 \\
\hline 3 & মুশারফ (Musharaf) & 0.05 & 0.01 & 0.86 & 0.02 & 0.06 \\
\hline 3 & কাশ্মীর (Kashmir) & 0.03 & 0.01 & 0.93 & 0.01 & 0.02 \\
\hline 3 & পাকিস্তান (Pakistan ) & 0.06 & 0.02 & 0.82 & 0.03 & 0.07 \\
\hline 3 & $\begin{array}{l}\text { नয়াদিল्লী (New } \\
\text { Delhi ) }\end{array}$ & 0.12 & 0.04 & 0.65 & 0.06 & 0.13 \\
\hline 3 & বর্ডার (Border) & 0.08 & 0.03 & 0.79 & 0.03 & 0.07 \\
\hline 4 & বিমা (Insurance) & 0.08 & 0.12 & 0.02 & 0.68 & 0.10 \\
\hline 4 & $\begin{array}{l}\text { স্বাস্থ্যবিমা ( Health } \\
\text { Insurance ) }\end{array}$ & 0.12 & 0.12 & 0.03 & 0.54 & 0.19 \\
\hline 5 & উত্পাদন (Creation) & 0.12 & 0.12 & 0.11 & 0.11 & 0.54 \\
\hline 5 & $\begin{array}{l}\text { নবীকরণযোগ্য (Able } \\
\text { of renewing ) }\end{array}$ & 0.10 & 0.10 & 0.05 & 0.15 & 0.50 \\
\hline 5 & $\begin{array}{l}\text { তাপমাত্রা } \\
\text { ( Temparature ) }\end{array}$ & 0.15 & 0.11 & 0.04 & 0.12 & 0.57 \\
\hline 5 & বিদ্যুত্ (Electricity) & 0.16 & 0.18 & 0.02 & 0.12 & 0.59 \\
\hline
\end{tabular}

$$
\text { Figure 1: The K-means clustering algorithm. }
$$

A set of initial cluster centers is necessary in the beginning. Each document is assigned to the cluster whose center is closest to the document. After all documents have been assigned, the center of each cluster is recomputed as the centroid or mean $\vec{\mu}$ (where $\vec{\mu}$ is the clustering coefficient) of its members (see Figure 1), that is $\vec{\mu}=\left(1 /\left|c_{j}\right|\right) \sum_{x \in c_{j}} \vec{x}$. The distance function is the cosine vector similarity function.

TABLE VI. FIVE CLUSTER CENTROIDS (MEAN $\vec{\mu}_{j}$ ).

Table VI gives an example of theme centroids from the $\mathrm{K}$ means clustering. Bold words in Theme column are cluster centers. Cluster centers are assigned by maximum clustering coefficient. For each theme word, the cluster from Table VI is still the dominating cluster. For example, "প্রশাসন" has a higher membership probability in cluster 1 than in other clusters. But each theme word also has some non-zero membership in all other clusters. This is useful for assessing the strength of association between a theme word and a topic. Comparing two members of the cluster 2, “কাশ্মীর" and "नয়াদিল्লী", it is seen that “নয়াদিল্লী" is strongly associated with cluster $2(\mathrm{p}=0.65)$ but has some affinity with other clusters as well (e.g., $\mathrm{p}=0.12$ with the cluster1). This is a good example of the utility of soft clustering. These non-zero values are still useful for calculating 
vertex weight during Theme Relational Graph (Described in Section V) generation.

\section{CONSTRUCTION OF DOCUMENT LEVEL THEME RELATIONAL GRAPH}

Representation of input text document(s) in the form of graph is the key to our design principle. The idea is to build a document graph $\mathrm{G}=<\mathrm{V}, \mathrm{E}>$ from a given source document $d \in D$. First, the input document $d$ is parsed and split into a number of text fragments (sentence) using sentence delimeters (Bengali sentence marker "I", “?” or "!"). At this preprocessing stage, text is tokenized, stop words are eliminated, and words are stemmed [13]. Thus, the text in each document is split into fragments and each fragment is represented with a vector of constituent theme words. These text fragments become the nodes $\mathrm{V}$ in the document graph.

The similarity between two nodes is expressed as the weight of each edge $\mathrm{E}$ of the document graph. A weighted edge is added to the document graph between two nodes if they either correspond to adjacent text fragments in the text or are semantically related by theme words. The weight of an edge denotes the degree of the relationship. The weighted edges not only denote document level similarity between nodes but also inter document level similarity between nodes. Thus to build a document graph $\mathrm{G}$, only the edges with edge weight greater than some predefined threshold value are added to $\mathrm{G}$, which basically constitute edges $\mathrm{E}$ of the graph $\mathrm{G}$.

The Cosine similarity measure has been used here. In cosine similarity, each document $\mathrm{d}$ is denoted by the vector $\vec{V}(d)$ derived from $\mathrm{d}$, with each component in the vector for each Theme words. The cosine similarity between two documents (nodes) $\mathrm{d} 1$ and $\mathrm{d} 2$ is computed using their vector representations $\vec{V}(d 1)$ and $\vec{V}(d 2)$ as equation (1) and (2) (Described in Section IV). Only a slight change has been done i.e. the dot product of two vectors $\vec{V}(d 1) \bullet \vec{V}(d 2)$ is defined as $\sum_{i=1}^{M} V(d 1) V(d 2)$. The Euclidean length of $\mathrm{d}$ is defined to be $\sqrt{\sum_{i=1 V_{i}}^{M} \rightarrow^{2}(d)}$ where $\mathrm{M}$ is the total number of documents in the corpus. Theme nodes within a cluster are connected by vertex, weight is calculated by clustering coefficient of those theme nodes. No inter cluster vertex are there. Cluster centers are interconnected with weighted vertex. The weight is calculated by cluster distance as measured by cosine similarity measure as discussed earlier.

To better aid our understanding of the automatically determined category relationships we visualized this network using the Fruchterman-Reingold force directed graph layout algorithm [16] and the NodeXL network analysis tool [17] ${ }^{6}$. A theme relational model graph drawn by NoddeXL is shown in figure 3 .

\footnotetext{
${ }^{6}$ Available from http://www.codeplex.com/NodeXL
}

\section{Thematic AFFinity Distance MEASUREMENT}

Generated Bengali thematic relational graph consist of four categories of POS (noun, adjective, adverb and verb) considered for present generation. In the generated Bengali thematic relational graph all the lexicons are connected with weighted vertex either directly or indirectly. Thematic affinity inference could be identified by network distance of any two nodes by calculating the distance in terms of weighted vertex. We computed the relevance of thematic nodes by summing up the edge scores of those edges connecting the node with other nodes in the same cluster. As cluster centers are also interconnected with weighted vertex so inter-cluster relations could be also calculated in terms of weighted network distance between two nodes within two separate clusters. As an example:

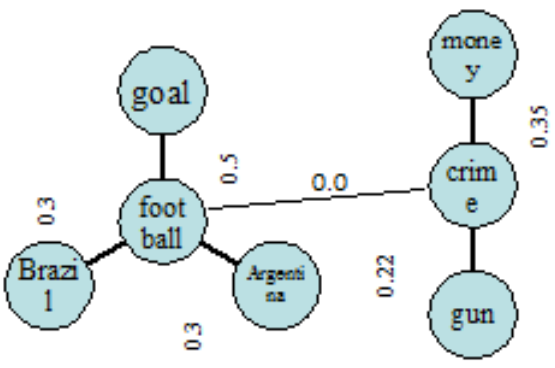

Figure 2: Thematic Affinity Graph

The thematic affinity inference from Figure 2 could be calculated as follows:

$$
\begin{aligned}
S_{d}\left(w_{i}, w_{j}\right) & =\frac{\sum_{k=0}^{n} v_{k}}{k} \\
& =\sum_{c=0}^{m} \frac{\sum_{k=0}^{n} v_{k}}{k} \times \prod_{c=0}^{m} l_{c}---(2)
\end{aligned}
$$

where $S_{d}\left(w_{i}, w_{j}\right)=$ thematic affinity distance between two themes $\mathrm{w}_{\mathrm{i}}$ and $\mathrm{w}_{\mathrm{j}}$. Equation (1) and (2) are for intra-cluster and inter-cluster thematic distance measure respectively. $k=$ number of weighted vertex between two themes $w_{i}$ and $w_{j}$. $\mathrm{V}_{\mathrm{k}}$ is the weighted vertex between two themes. $\mathrm{m}=$ number of cluster centers between two theme nodes. $l_{c}$ is the distance between cluster centers between two theme nodes.

For illustration of present technique let take an example:

$$
\begin{gathered}
(\text { Argentina, goal })=\frac{0.5+0.3}{2}=0.4 \\
(\text { Gun, goal })=\left(\frac{0.22}{1}+\frac{0.5}{1}\right) \times 0.0=0
\end{gathered}
$$

It is evident from the previous example that the score based thematic distance can better illustrate topical relation among documents. 


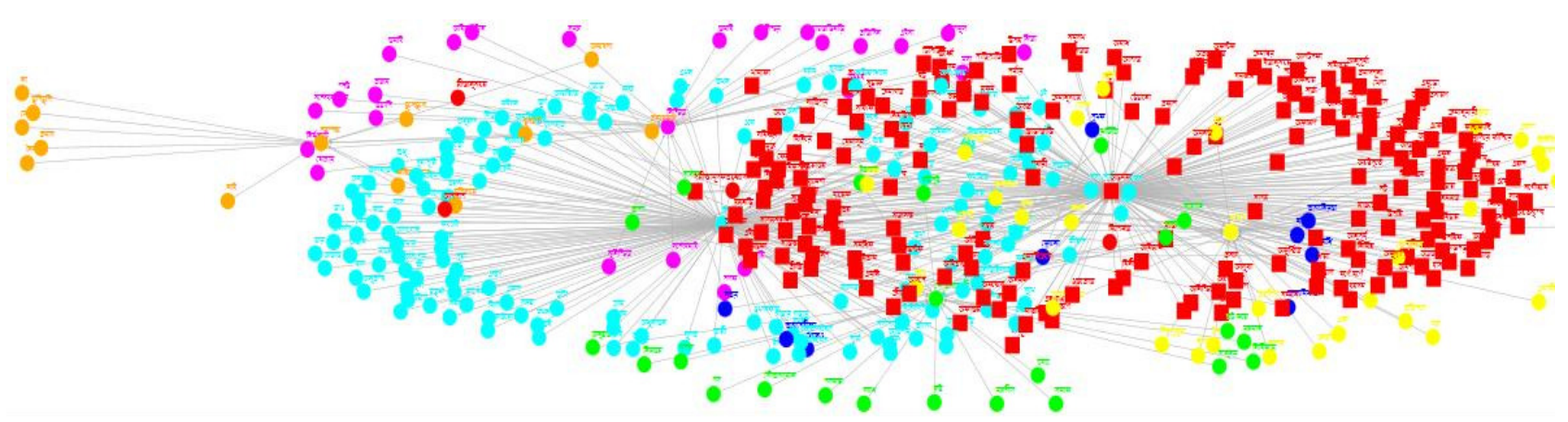

Figure 1. Document Level Theme Relational Graph by NodeXL.

\section{SUMMARIZATION SYSTEM}

Present system is an extractive opinion summarization system for Bengali. In the previous sections, we described how to identify theme clusters that relates to different shared topics and subtopics, from a given input document set. But identifying those clusters is not only a step toward generating document level opinionated news summary rather there is another major step is to extract thematic sentences from each theme cluster that reflects the contextual concise content of the current theme cluster. Extraction of sentences based on their importance in representing the shared subtopic (cluster) is an important issue and it regulates the quality of the output summary. We have used Information Retrieval (IR) based technique to identify the most "informed" sentences from any cluster and it can be termed as IR based cluster center for that particular cluster. With the adaptation of ideas from page rank algorithms [18], it can be easily observed that a text fragment (sentence) in a document is relevant if it is highly related to many relevant text fragments of other documents in the same cluster. Since, in our document graph structure, the edge score reflects the correlation measure between two nodes, it can be used to identify the most salient/informed sentence from a sentence cluster.

\section{TABLE VII. CANDIDATE SENTENCES}

\begin{tabular}{|c|c|}
\hline Candidate Sentence & $\begin{array}{c}\text { IR } \\
\text { Score }\end{array}$ \\
\hline $\begin{array}{l}\text { মহম্মদ আমিনের মতো পলিটব্যুরোর 'নবীনতম' } \\
\text { সদস্যকেও কিন্তু বয়সের দিক হইতে নবীন ভাবা কঠিন। }\end{array}$ & 151 \\
\hline $\begin{array}{l}\text { এবার চিন্তা আরওএকটু বেশি, কারণ এই মূল্যবৃদ্ধির পিজনে } \\
\text { যেমন দেশের ভিতরে ভিনিসপত্রের জোগান কমে যাওয়া আছে, } \\
\text { তেমনই আছে আন্তর্ডাতিক বাজারে মূল্যবৃদ্ধির প্রবণতা। }\end{array}$ & 167 \\
\hline $\begin{array}{l}\text { স্বাধীনতার পর যাট বছ্নর গত হইল, এখনও প্রায় সকল } \\
\text { সরকারি পরিকল্পনার পিজ্ননে এই একটিই ভাবাদর্শ কাজ } \\
\text { করে: বিভিন্ন তোটব্যাঙ্ককে তুষ্ট করিয়া যেন তেন প্রকারেণ } \\
\text { নিজেদের দলীয় স্থিতি নিশ্চিত করা। }\end{array}$ & 130 \\
\hline
\end{tabular}

We computed the relevance of a node/sentence by summing up the edge scores of those edges connecting the node with other nodes in the same cluster. Then the nodes are given rank according to their calculated relevance scores and the top ranking sentences is selected as the candidate sentence representing the opinion summary. For example four such candidate sentences are shown in Table VII. The words in bold are the theme words based on those theme words the sentences are extracted.

Another issue that is very important in summarization is sentence ordering so that the Output summary looks coherent. Once all the relevant sentences are extracted across the input documents, the summarizer has to decide in which order to present them so that the whole text makes sense for the user. We prefer the original order of sentences as they occurred in original document.

\section{EXPERIMENTAL RESULT}

The evaluation result of the CRF-based Theme Detection task for Bengali is presented in Table VIII. The result presented individually for every annotators and the overall result of the system.

TABLE VIII. RESULTS OF CRF-BASED THEME IDENTIFIER

\begin{tabular}{|c|c|c|c|c|c|}
\hline \multirow{4}{*}{ 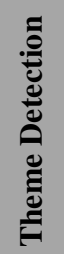 } & Metrics & $\mathbf{X}$ & $\mathbf{Y}$ & $\mathbf{Z}$ & Avg \\
\hline & Precision & $87.65 \%$ & $85.06 \%$ & $78.06 \%$ & $83.60 \%$ \\
\hline & Recall & $80.78 \%$ & $76.06 \%$ & $72.46 \%$ & $76.44 \%$ \\
\hline & F-Score & $84.07 \%$ & $80.30 \%$ & $75.16 \%$ & $79.85 \%$ \\
\hline
\end{tabular}

The evaluation result of subjective sentence identification of the system for opinion summary is in the Table IX.

TABLE IX. FINAL RESULTS SUBJECTIVE SENTENCE IDENTIFICATION FOR SUMMARY.

\begin{tabular}{|c|c|c|c|c|c|}
\hline \multirow{4}{*}{ 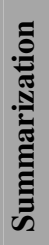 } & Metrics & $\mathbf{X}$ & $\mathbf{Y}$ & $\mathbf{Z}$ & Avg \\
\hline & Precision & $77.65 \%$ & $67.22 \%$ & $71.57 \%$ & $72.15 \%$ \\
\hline & Recall & $68.76 \%$ & $64.53 \%$ & $68.68 \%$ & $67.32 \%$ \\
\hline & F-Score & $72.94 \%$ & $65.85 \%$ & $70.10 \%$ & $69.65 \%$ \\
\hline
\end{tabular}




\section{CONCLUSION}

In this work we have reported our work on single-document opinion summarization for Bengali. The novelty of the proposed technique is in topic based document-level theme relational graphical representation. According to best of our knowledge this is the first attempt on opinion summarization for Bengali. The approach presented here is unique in every aspect as in literature and for new language Bengali.

Our next research target is to generate a hierarchical cluster of theme words with time-frame relations. Time-frame relations could be useful for time wise opinion tracking.

\section{REFERENCES}

[1] Paul Resnick, Ko Kuwabara, Richard Zeckhauser, and Eric Friedman. Reputation systems. Communications of the Association for Computing Machinery (CACM), 43(12):45-48, 2000. ISSN 0001-0782.

[2] Lun-Wei Ku, Li-Ying Li, Tung-Ho Wu, and Hsin-Hsi Chen. Major topic detection and its application to opinion summarization. In Proceedings of the SIGIR, pages 627-628, 2005. Poster paper.

[3] Liang Zhou and Eduard Hovy. On the summarization of dynamically introduced information: Online discussions and blogs. In AAAI-CAAW, pages 237-242, 2006

[4] Giuseppe Carenini, Raymond Ng, and Adam Pauls. Multi-document summarization of evaluative text. In Proceedings of the European Chapter of the Association for Computational Linguistics (EACL), pages $305-312,2006$

[5] M. Hu and B. Liu. 2004a. Mining and summarizingcustomer reviews. In Proc. of the 10th ACMSIGKDD Conf., pages 168-177, New York, NY, USA. ACM Press.

[6] Yukiko Kawai, Tadahiko Kumamoto, and Katsumi Tanaka. Fair News Reader: Recommending news articles with different sentiments based on user preference. In Proceedings of KES, number 4692 in Lecture Notes in Computer Science, pages 612-622, 2007.

[7] A. Das and S. Bandyopadhyay (2009a). Subjectivity Detection in English and Bengali: A CRF-based Approach., In Proceeding of ICON 2009, Decem-ber 14th-17th, 2009, Hyderabad.

[8] A. Das and S. Bandyopadhyay (2010a). SentiWordNet for Bangla., In Knowledge Sharing Event-4: Task 2: Building Electronic Dictionary , February 23th to 24th, 2010, Mysore.

[9] Vasileios Hatzivassiloglou and Janyce Wiebe. Effects of adjective orientation and gradability on sentence subjectivity. In Proceedings of the International Conference on Computational Linguistics (COLING), pages 299-305, 2000.

[10] Paula Chesley, Bruce Vincent, Li Xu, and Rohini Sri-hari. Using verbs and adjectives to automatically classify blog sentiment. In AAAICAAW, pages 27-29, 2006.

[11] Tetsuya Nasukawa and Jeonghee Yi. Sentiment anal-ysis: Capturing favorability using natural language processing. In Proceedings of the KCAP, pages 70-77, 2003.

[12] A. Das and S. Bandyopadhyay. Theme Detection an Exploration of Opinion Subjectivity. In Proceeding of Affective Computing \& Intelligent Interaction (ACII 2009b).

[13] A. Das and S. Bandyopadhyay (2010b). Morphologi-cal Stemming Cluster Identification for Bangla., In Knowledge Sharing Event-1: Task 3: Morphologi-cal Analyzers and Generators, January, 2010, Mysore.

[14] Jardine, N. and van Rijsbergen, C. J. (1971). The use of hierarchic clustering in information retrieval. Information Storage and Retrieval, 7, 217-240.

[15] Willerr, P. (1988). Recent trends in hierarchic document clustering: A critical review. Information Processing and Management, 24(5), 577597.

[16] Thomas M. J. Fruchterman and Edward M. Reingold. 1991. Graph drawing by force-directed placement. Software: Practice and Experience, 21(11):1129-1164.
[17] Marc Smith, Ben Shneiderman, Natasa Milic-Frayling, Eduarda Mendes Rodrigues, Vladimir Barash, Cody Dunne, Tony Capone, Adam Perer, and Eric Gleave. 2009. Analyzing (social media) networks with NodeXL. In C\&T '09: Lecture Notes in Computer Science. Springer.

[18] Lawrence Page, Sergey Brin, Rajeev Motwani, and Terry Winograd. 1998. The PageRank Citation Ranking: Bringing Order to the Web. Technical report, Stanford Digital Library Technologies Project. 